\title{
Spin Asymmetries and Helicity Amplitudes in Photo-Production from Polarized Neutrons
}

\section{A.M. Sandorfi}

Received: date / Accepted: date

\begin{abstract}
Pseudoscalar meson photoproduction experiments lead the search for an expanded $N^{*}$ spectroscopy that is required by lattice solutions of QCD. Extensive data have been collected with proton targets that can potentially over-determine the production amplitude and allow a search for weak resonances. In contrast, data on photoproduction from neutrons is drastically limited. Both are needed since the $\gamma p N^{*}$ and $\gamma n N^{*}$ photo-couplings to $I=1 / 2$ states are different and provide complementary information on the mechanisms for resonance excitation. The main subtleties in experiments with an effective polarized neutron target are reviewed. The considerable impact of new polarization data on multipole analyses are discussed, using the example of recent $\pi^{-} p$ measurements of the beam-target helicity asymmetry $(E)$ from polarized $H D$ in CLAS at Jefferson Lab. New partial wave analyses incorporating these data have found significant changes in helicity amplitudes of some established resonances, and signatures of weak resonances that previously were observed only in hyperon decay channels.
\end{abstract}

Keywords photoproduction · polarized neutrons · helicity amplitudes

PACS 25.20.Lj $\cdot$ 13.88.+e $\cdot$ 13.60.Le $\cdot 14.20 . \mathrm{Gk}$

Work supported by the US Department of Energy, Office of Nuclear Physics Division, under contract DE-AC05-06OR23177 under which Jefferson Science Associates operate Jefferson Laboratory.

A.M. Sandorfi

Thomas Jefferson National Accelerator Facility, Newport News, VA 23606 USA

Tel.: +1 7572695457

Fax: +1 7572696418

E-mail: sandorfi@JLab.org 


\section{The $N^{*}$ spectrum and meson photo-production}

In the evolution of the early universe, at about a microsecond after the initial singularity, a dramatic chiral phase transition occurred. Chiral symmetry was broken, color confinement emerged, current quarks acquired mass from clouds of gluons as they cooled to form effective constituent objects [1], and these combined into colorless baryons with internal excitations. For that brief epoch, excited nucleons $\left(N^{*} \mathrm{~s}\right)$ flooded space.

Quark models (QM), which captured the symmetries of QCD without solving its Lagrangian, predicted an extensive array of excited nucleon states [2]. Until recently, only a modest fraction of predicted QM resonances had been observed with three- or four-star status (as designated by the Particle Data Group), despite the fact that the full complement of QM states were required to account for the baryon pressure during the chiral phase transition [3]. Recently, direct solutions of QCD on a Lattice (LQCD) confirmed the QM expectation of an extensive $N^{*}$ spectrum [4].

The key goals of the $N^{*}$ spectroscopy program in CLAS are to elucidate the structure of the $N^{*}$ states that have been observed and to find the ones that so far have remained elusive. Up to a decade ago, information on excited nucleon levels has come almost exclusively from $\pi \mathrm{N}$ reactions. These are described by two complex spin-dependent amplitudes, and so require a minimum of three independent measurements to describe the process, within a phase. There are four possible $\pi \mathrm{N}$ observables - the cross section $(\sigma)$, a recoil polarization $(P)$ and two double-polarization asymmetries $(R$ and $A$ ). While there are extensive data on $\sigma$ and $P$, measurements of $R$ and $A$ are almost non-existent, a mere 30 points for each at energies above the first resonance, the $\Delta(1232) 3 / 2+$. As a result, the $\pi \mathrm{N}$ reaction amplitudes are under-determined, which renders the search for weak resonances highly problematic.

The CLAS spectroscopy program is focused on meson photo-production. Due to the additional spin of the photon, photo-production requires four complex spin-dependent amplitudes to specify the reaction, and a minimum of 7 measurements (or in practice, 8 to avoid ambiguities [5]). However, there are 16 possible observables that can be measured, each in two independent ways [6] and, at least for proton targets, data on all have been collected with CLAS and are in various stages of analysis.

When comparing photo-production processes to a predicted spectrum, complications enter through what are usually called dressing of the interaction vertices. A resonance is associates with a pole in an s-channel diagram, such as in Fig. 1. But considerable details are buried in the vertices. The higher energies of the missing $N^{*}$ levels are above thresholds for many decay channels. Hyperon channels, for example, can be either produced directly (eg. $\gamma p \rightarrow K \Lambda$ ) or reached through various two-step processes (eg. $\gamma p \rightarrow \pi N \rightarrow K \Lambda$ ). As a result, calculating the strong vertex of Fig. 1 becomes a multi-channel, multiresonance problem, with Unitarity requirements connecting all possible channels. Such coupled-channel dressings of the strong vertex essentially determine the spectral properties of $N^{*}$ resonances - their mass/pole positions and 


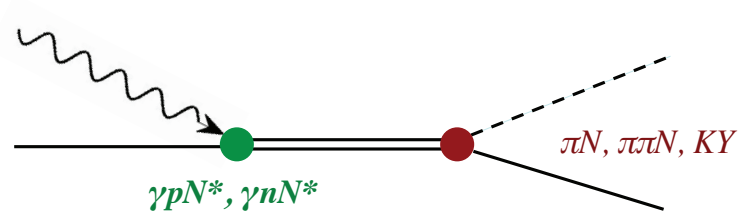

Fig. 1 An s-channel pole excited through an electromagnetic vertex, with subsequent decay through the strong vertex.

widths. (A prime illustration is given in ref. [7], where channel couplings are shown to dramatically change the pole position of the $P_{11}$ Roper resonance.)

The electromagnetic vertex of Fig. 1 is dressed with a cloud of spin zero mesons $(\pi, \eta, \kappa)$. The photon can attach, for example, to a nucleon that is off-shell due to the presence of a virtual pion loop, or it can attach to the pion itself, etc. Such mesonic dressings of the electromagnetic vertex affect the dynamical properties of resonance excitation, the $\gamma N N^{*}$ vertex couplings (and their $Q^{2}$ evolution), but do not affect spectral properties. (The $N(1675) 5 / 2^{-}$ resonance illustrates these complexities. Standard single-quark excitations to this state are forbidden by selection rules, but the measured photo-couplings are an order of magnitude larger than QM expectations [8].)

The photon vertex of Fig. 1 is further complicated by the fact that the electromagnetic interaction does not conserve isospin. For example, the amplitude for the $N(\gamma, \pi)$ reaction factors into distinct isospin components, $\mathcal{A}_{\left(\gamma, \pi^{ \pm}\right)}=$ $\sqrt{ } 2\left\{\mathcal{A}_{p / n}^{I=1 / 2} \mp 1 / 3 \mathcal{A}^{I=3 / 2}\right\}$. While the excitation of $I=3 / 2 \Delta^{*}$ states can be entirely determined from proton target data, measurements with both neutron and proton targets are required to deduce the isospin $I=1 / 2$ amplitudes, and separate $\gamma p N^{*}$ and $\gamma n N^{*}$ couplings. Generally, the latter are very poorly determined, due to the paucity of neutron reaction data.

\section{New experiments with polarized neutrons}

To improve the determination of neutron photo-couplings and to expand the search for as yet unidentified $N^{*}$ s, the E06-101 experiment at Jefferson Lab, the g14 run with the CEBAF Large Acceptance Spectrometer (CLAS) in Hall B [9], has been recently carried out. Data on a number of previously unmeasured observables are emerging. First results on the beam-target double polarization asymmetry,

$$
E=\frac{1}{P_{\gamma} P_{T}} \frac{\sigma_{A}-\sigma_{P}}{\sigma_{A}+\sigma_{P}}
$$

have recently been reported for the quasi-free reaction $\gamma n(p) \rightarrow \pi^{-} p(p)$, through the $N^{*}$ resonance region [10]. This beam-helicity asymmetry is measured with beam $\left(P_{\gamma}\right)$ and target polarizations $\left(P_{T}\right)$ anti-parallel $(A)$ and parallel $(P)$ to the beam momentum, respectively. (We use the sign convention as detailed in Ref. [11].) 

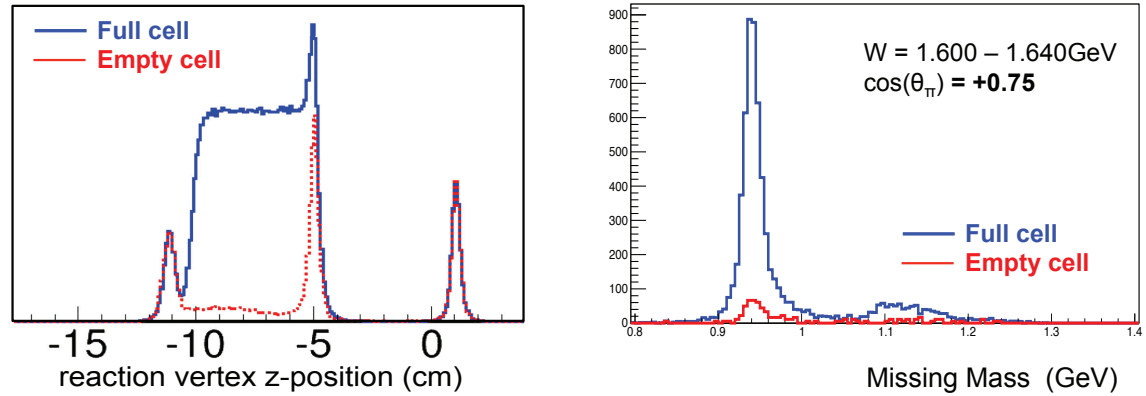

Fig. 2 Reaction vertex position (left panel) along the beam direction $(z)$, reconstructed by tracking $\pi^{-}$and $p$ in CLAS, shown for equivalent-flux data from full (blue) and empty (red) targets. Beam entrance and exit windows generate peaks at -11 and $-5 \mathrm{~cm}$, respectively. A target-independent foil in the cryostat generates the peak at $+1 \mathrm{~cm}$. Typical missing mass $(M M)$ distribution (right panel) in a single kinematic bin. A requirement of $M M<1.05$ selects the $\pi^{-} p(p)$ final state.

Here, we highlight some of the important considerations encountered with neutron targets, using the example of the CLAS g14 experiment, and leave details regarding the yield extraction for the final state of interest to ref. [10].

\subsection{Polarized target}

The CLAS experiment, using polarized deuterium for an effective neutron target, has been carried out with solid hydrogen-deuteried $(H D)$, polarized and in a frozen-spin state. A typical reconstruction of the target vertex is shown in the left panel of Fig. 2. (The small Empty cell yield between the beamentrance and -exit windows comes from thin $\mathrm{Al}$ cooling wires that are needed during the polarization process.) A missing mass distribution, reconstructed for the $\gamma n(p) \rightarrow \pi^{-} p(p)$ reaction, is shown in the right panel for one kinematic bin. While the operation of this target is quite complex, it has the distinct advantages of having very little background, no dilution from unpolarizable neutrons (other than in the target cell, which can be subtracted away), and inbeam polarization lifetimes $\left(T_{1}\right)$ on the order of years. The polarizations of $H$ and $D$ are monitored during the experiment with Nuclear Magnetic Resonance $(N M R)$ methods, and the spin orientations are flipped occasionally. Further details are given in refs. [12,13].

\subsection{Restricting kinematics to create an effective neutron target}

Since there are no free neutron targets, a requirement common to the analysis of all experiments with deuterium targets is the selection of events for which the neutron in deuterium is as close to free as possible. The key parameter is the neutron momentum in the deuteron, or equivalently the reconstructed 


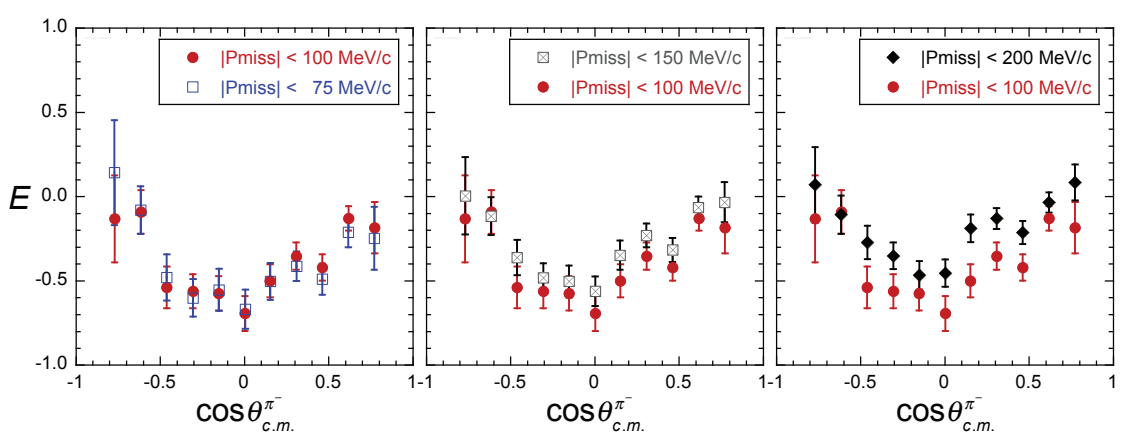

Fig. 3 Angular distributions of the $E$ asymmetry in the $\gamma n \rightarrow \pi^{-} p$ reaction at $W=1580$ $\mathrm{MeV}$, deduced from the $\gamma n(p) \rightarrow \pi^{-} p\left(p_{\text {miss }}\right)$ process with different limiting requirements on the reconstructed momentum of the missing proton. The standard requirement of $\left|P_{\text {miss }}\right|<$ $0.10 \mathrm{GeV} / \mathrm{c}$ from ref. [10] is shown as red circles in all three panels. Increasing the acceptance window to 0.15 and to $0.20 \mathrm{GeV} / \mathrm{c}$ results in the grey squares and the black diamonds in the center and left panels, respectively. Decreasing the window further to $\left|P_{\text {miss }}\right|<0.075$ $\mathrm{GeV} / \mathrm{c}$ results in the open-blue squares of the right panel [14].

momentum of the undetected (spectator) proton, $P_{\text {miss }}$. We have found it essential to determine the optimum threshold from the data itself. Studies with individual kinematic bins have shown a dilution of the $E$ asymmetry when the maximum $\left|P_{\text {miss }}\right|$ limit is increased above $0.1 \mathrm{GeV} / \mathrm{c}$, but no statistically significant change for smaller values. This is obvious in Fig. 3 where angular distributions with different thresholds are plotted for one energy bin. An average over the full kinematic range has been presented in ref. [10] as a function of missing momentum. Again, the average is stable below $0.1 \mathrm{GeV} / \mathrm{c}$ but rises significantly at higher $\left|P_{\text {miss }}\right|$.

A common limiting value for $P_{\text {miss }}$ that has been used in many analyses with deuterium targets in the literature is $0.2 \mathrm{GeV} / \mathrm{c}$. This is often viewed as an appealing compromise, in that it removes a long high-momentum tail while keeping most of the missing momentum distribution. The more restrictive requirement of $\left|P_{\text {miss }}\right|<0.1 \mathrm{GeV} / \mathrm{c}$ costs about a factor of two in statistics. Nonetheless, as evident in Fig. 3, this is absolutely essential if one is to associate the results with an effectively free neutron. One must be wary of unsubstantiated hopes that final-state-interaction effects, while significant in cross section measurements, might generally be small in polarization asymmetries. From $g 14$ analyses of different asymmetries, the only general rule we have found is that there is no general rule. The safe window of the $P_{\text {miss }}$ distribution that will provide reliable information on a free neutron is observable-dependent and must be studied for each case.

2.3 The $D$-state of the deuteron and spin rotations of the moving neutron

While the neutron and proton within the deuteron are mostly in a relative $S$-state with spins parallel, the assignment of the neutron's polarization can 

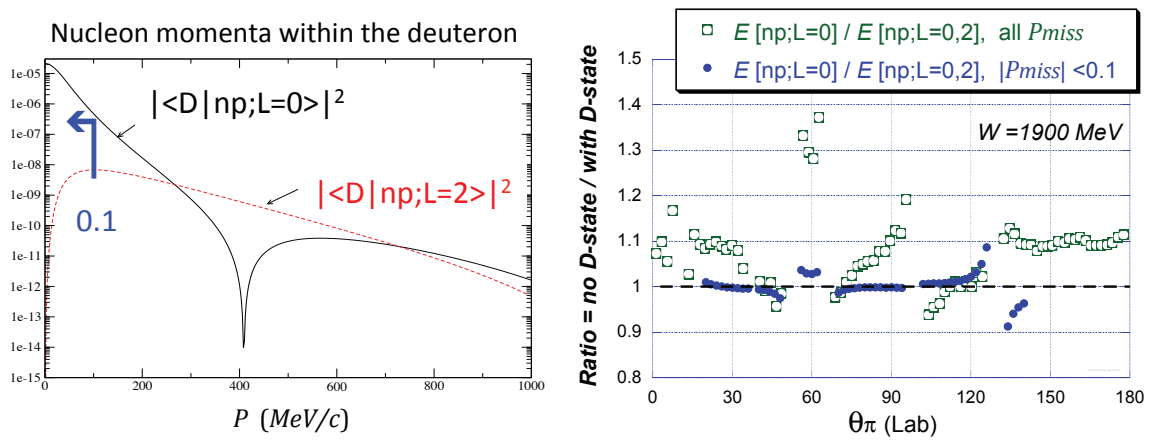

Fig. $4 S$ and $D$ state probabilities within the deuteron (left panel), from the calculation of [15]. The ratio of calculations for the beam-target $E$ asymmetry in the $\pi^{-} p$ final state, without and with the inclusion of the deuteron's $D$-wave component, is shown in the right panel for different requirements on the momentum of the spectator proton - see text. (The slight deviations from unity in the solid blue circles near $60^{\circ}$ and $130^{\circ}$ occur when the asymmetry crosses zero, where small variations in the calculations are amplified.)

be complicated by the $D$-wave component in the deuteron wavefunction. (The latter is not strictly an observable. It is only necessary that it be treated in a self-consistent way within an $N N$ potential. Typical values range between 3-6 $\%$.) The $D$-wave fraction is momentum dependent, as seen in the left panel of Fig. 4. While insignificant at low nucleon momenta, it can even dominate at higher momenta.

The effect of the deuteron's $D$-state on the $E$ asymmetry has been studied using an impulse approximation within the formulation of Ref. [15], extended to include all relativistic transformations of the spin of the moving neutron [16]. The right panel of Fig. 4 shows the ratios of two calculations of the $E$ asymmetry, without and with the inclusion of the deuteron's $D$-state. While the $E$ asymmetry can be significantly altered whenever high spectator momenta are present, the effect of the $D$-wave component is suppressed to negligible levels by the $\left|P_{\text {miss }}\right| \leq 0.1 \mathrm{GeV} / \mathrm{c}$ requirement. Thus, with the latter requirement, the polarization of the effective neutron target is just the polarization of the deuteron, as determined from experimental $N M R$.

When considering the $\gamma n \rightarrow \pi^{-} p$ reaction, the center of mass frame is reached by a boost along the momentum of the neutron within the deuteron. When boosting a baryon's spin projections, a Wigner-Thomas precession of the spin can arise due to the fact that boosts and rotations do not commute [17]. However, this effect is negligible in the case of the $E$ asymmetry, because the boost direction is along the neutron momentum. This has been verified by explicit calculation for spin asymmetries associated with the target polarization; but the corrections can become quite large when a recoil polarization is involved [18]. 


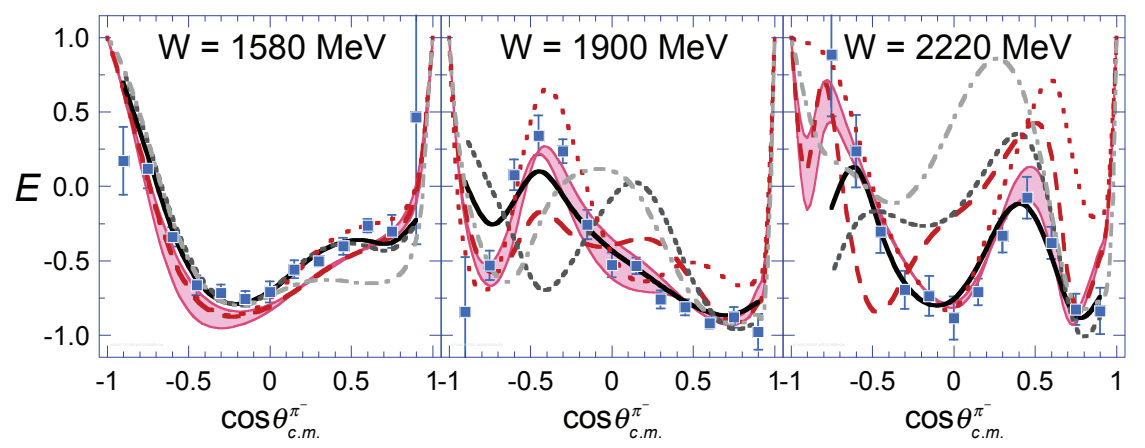

Fig. 5 Beam-target $E$ asymmetries (blue squares) from the CLAS $g 14$ experiment for a sample of three invariant mass bins [10]. Shown with these are recent PWA fits that include these data: solid red curves from SAID [19], with shaded bands indicating variations across the energy bin; solid black lines from BnGa [20]. Also shown are previous PWA solutions that did not include the present data set in the multipole search: red-dotted curves from SAID[CM12], based on all data up to 2012 [21]; red-dashed curves from SAID[AS25], including all previously published data; grey dot-dashed curves from BnGa[2014-02], based on all data up to 2014 [22]; black short-dashed curves from a BnGa PWA using all previously published data. (See text.)

\section{Beam-target helicity asymmetries and Partial Wave Analyses}

The beam-target double-polarization asymmetries of Eg. 1 have been reported recently in ref [10] for the $\gamma n \rightarrow \pi^{-} p$ reaction. A sample of the measured asymmetries are shown here in Fig. 5 as blue squares for three invariant mass bins. These are the first results for this observable and demonstrate the impact of new asymmetry data on as yet under-determined amplitudes. The predictions from previously published Partial Wave Analyses (PWA), the red-dotted and grey dash-dotted curves in the figure, are close to the $E$ data at low energies, but they become wildly disparate for $W$ above about $1800 \mathrm{MeV}$ [10].

New PWA of $\pi$ photoproduction have been carried out, augmenting the neutron data base with these new $E$ asymmetries. New PWA from the George Washington University Data-Analysis group (SAID) [19], and new PWA from the Bonn-Gatchina (BnGa) group [20], are shown as solid red and solid black curves in Fig. 5, respectively. Both provide very good representations of the new $E$ data. PWA combine results from many experiments at different energies, and this results in varying degrees of sensitivity to energy and angle. This is illustrated by the red bands whose width indicates the SAID variation across the energy bin.

While data on other observables have emerged since the last published PWAs, these new CLAS $g 14 E$ asymmetries alone have a significant impact on multipole solutions. To illustrate their explicit effect, we have included in Fig. 5 predictions from more recent PWA that include all currently published data [23] (but exclude the new $\pi^{-} p E$ asymmetries); these are shown as the red dashed and black short-dashed curves. In the higher invariant mass range, 
these are drastically different from the measured beam-target asymmetry results.

\subsection{The benefit of different PWA approaches}

New PWA can be examined for information on resonances and their photocouplings. However, the current data base for the $\gamma n \rightarrow \pi^{-} p$ reaction is still extremely limited: $d \sigma$ (2322 kinematic points), $\Sigma$ (315 points), $T$ (105 points), $P$ (75 points), and now $E$ (263 points) [24]. As discussed in Sec. 1, these alone are insufficient to remove ambiguities in multipole amplitudes. This being the case, it is fortuitous that complementary PWA are available which search for potential $N^{*}$ resonances in very different ways.

In any PWA, the transition amplitude takes the general form,

$$
T_{\alpha \gamma}=\sum_{\sigma} \frac{K_{\sigma \gamma}}{(1-i c K)_{\alpha \sigma}},
$$

where the sum extends over some set of hadronic states, with photo-couplings $K_{\sigma \gamma}$. The poles in the denominator generate the resonances.

In the SAID approach [21], the denominators in Eq. 2 are fixed by simultaneous fits to $\pi N \rightarrow \pi N$ and $\pi N \rightarrow \eta N$ reaction data. Then in a subsequent step, the numerators are parameterized as polynomials in the invariant mass, $K(W)$, and fitted to photoproduction data. Thus, all poles are determined entirely by $\pi N$ reactions, and photoproduction data can be used only to determine photo-couplings.

In contrast, the BnGa approach is a simultaneous fit of both $\pi N$ and $\gamma N$ reactions [25]. The $\pi N \rightarrow \pi N$ process is fitted to the SAID amplitudes, and trial resonances are added sequentially to each partial wave and compared to photo-production data. The overall solution minimizes $\chi^{2}$ over all channels.

These two approaches are sufficiently different that it is reasonable to attach a higher level of significance when they agree!

\subsection{Helicity amplitudes and photo-couplings}

The strength of the $\gamma n N^{*}$ coupling at the photon vertex of Fig. 1 reflects the excitation mechanism. Formally, this coupling is the (complex) residue from an analytic continuation of the amplitude to the pole in the complex energy plane. Conceptually, it is easier to picture when the transition amplitude of Eq. 2 is parametrized with a Briet-Wigner form, in which the numerator is replaced with a product of partial widths for formation and decay of a resonance, $A^{h} \cdot g_{\sigma}$, respectively. In the helicity basis, the $A^{h}$ are just the photo-couplings for the two different choices of entrance channel helicity. While there is no a priori reason why the magnitude of the residue need be the same as the (real) $A_{B W}^{h}$ deduced from a Breit-Wigner fit (unless the pole is very close to the real axis), in fact for most $N^{*}$ s they are, and for the cases discussed here the two 
values are indistinguishable. Since the physical significance of the phases of the complex helicity amplitudes is illusive, we will discuss only their magnitudes.

\subsection{Revised photo-couplings of established resonances}

The new PWA fits of Fig. 5 have resulted in significant changes to deduced resonance parameters. As expected, the $I=3 / 2$ partial waves, which can be determined entirely from proton target data, have remained essentially unaltered, while various $I=1 / 2$ waves have changed substantially. Agrand graphs of the imaginary parts of multipoles plotted against their real parts provide a useful way of visualizing changes. When plotting multipoles at sequentially increasing values of invariant mass, $\mathrm{W}$, every resonance produces a counterclockwise rotating loop in an Argand plot. (Not every loop is a resonance, since interfering backgrounds can alter the phase motion. But a loop is an indicator of a region deserving further study.) As examples, in Fig. 6 we show Argand plots of the $\left(L^{\pi N}\right)_{I J}(n / p) E / M=P_{13} n M$ (left panel) and $G_{17} n M$ (right panel) partial waves from SAID analyses. The red points show the last published SAID solution from 2012 [21]. The light-green points result from new fits which include all subsequently published data up to January of 2017 (with the exception of the CLAS $g 14 E$ asymmetries), and the dark green points are multipoles from a fit to all data, including the $g 14 E$ asymmetries [10]. The latter reveal the expected counter-clockwise phase motion near the $N(1720) 3 / 2^{+}$and $N(2190) 7 / 2^{-}$resonances, each ranked four star by the Particle Data Group [26]. The locations of their corresponding pole positions are indicated by open-black arrows.

The new deduced values for the corresponding helicity amplitudes are listed in the third column of Table 1 . These couplings for the $N(1720) 3 / 2^{+}$resonance have changed appreciably compared to the the last published solution (second column). Recently, a new large data set of $\gamma n \rightarrow \pi^{-} p$ cross sections, obtained from data taken during the CLAS $g 13$ running period, has been published [28]. A fit to those results, combined with all data published up to January of 2017 (but excluding the $g 14 E$ data), has led to the helicity couplings listed in the fourth column of Table 1. Finally, the last column gives the results of a combined fit to all currently available data on the $\gamma n \rightarrow \pi^{-} p$ reaction, including both $g 13$ and $g 14$ results from CLAS. The addition of different combinations of the new data sets leads to stable values for the helicity amplitudes, within uncertainties, all of which are significantly different from the previous SAID[SN11] results.

In previously published SAID analyses [27,21], the $G_{17}$ wave had been too small to extract couplings for the $N(2190) 7 / 2^{-}$resonance. The new $g 14$ data require a much larger multipole component. For comparison, the recent BnGa coupled-channel PWA, solid black curves of Fig. 5, finds couplings of $A_{n}^{1 / 2}=+30 \pm 7$ and $A_{n}^{3 / 2}=-23 \pm 8$ (in the same units) for this state [10, 24]. Given the differences in approach, the agreement on the $A_{n}^{3 / 2}$ helicity amplitude is important. 


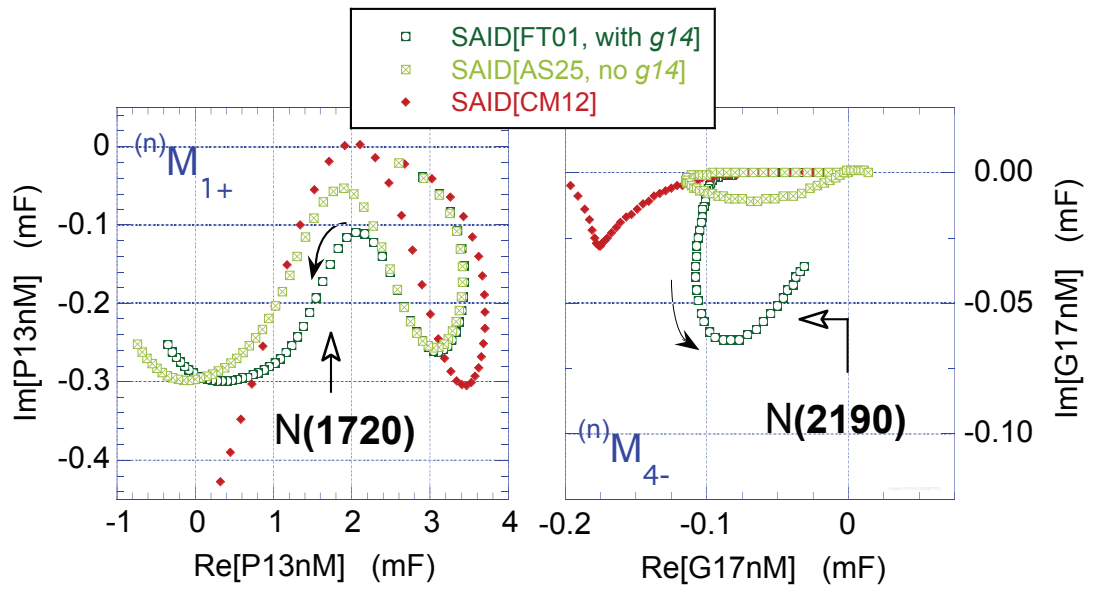

Fig. 6 Argand plots of the SAID PWA solutions of the $P_{13} n M$ (left) and $G_{17} n M$ (right) multipoles from $\pi$-threshold to $W=2300 \mathrm{MeV}$. Solid arrows indicate increasing $W$. As in the legend, red diamonds are the on-line versions [21], light-green crossed squares are fitted to all previously published data, and dark-green circles augment these with the new $E$ asymmetries from ref. [10].

Table $1 \gamma n N^{*}$ helicity amplitudes from the SAID analysis. The values in the columns from left to right reflect the addition of new data sets to the PWA fits. The solutions are from refs. [27] (SN11), [10] (FT01), [28] (MA27), and [29] (TS21) (See Text for further details.)

\begin{tabular}{lcccc}
\hline$\left(10^{-3} \mathrm{GeV}^{-1 / 2}\right)$ & Last Published & $+g 14(E)$ & $+g 13(\sigma)$ & $+g 14(E)+g 13(\sigma)$ \\
PWA solution: & SN11 & FT01 & MA27 & TS21 \\
\hline$A_{n}^{1 / 2}:$ & & & & \\
$N(1720) 3 / 2^{+}$ & $-21 \pm 4$ & $-9 \pm 2$ & $-16 \pm 6$ & $-15 \pm 5$ \\
$N(2190) 7 / 2^{-}$ & - & $-6 \pm 9$ & & $-16 \pm 5$ \\
& & & & \\
$A_{n}^{3 / 2}:$ & $-38 \pm 7$ & $+19 \pm 2$ & $+17 \pm 5$ & $+13 \pm 4$ \\
$N(1720) 3 / 2^{+}$ & - & $-28 \pm 10$ & & $-35 \pm 5$ \\
$N(2190) 7 / 2^{-}$ & & & & \\
\hline
\end{tabular}

3.4 Signals of weak resonances enhanced by polarization data

An expanded region of the Argand plot for the $P_{13} n M$ multipole from the recent $\mathrm{BnGa}$ analysis that has been fitted to the CLAS $g 14 E$ data is shown in Fig. 7. The pole position of the $N(1720) 3 / 2^{+}$resonance is indicated. While this occurs within a characteristics counter-rotating loop, the proximity of the $\rho$ threshold complicates this coupled-channel analysis and leads to large uncertainties in the helicity amplitudes [24] - see Table 2 below. The pole position of an $N(1900) 3 / 2+$ is indicated in the figure. While this is now classified as a three-star resonance [26], it couples strongly to hyperon channels rather than to $\pi N$. At the high energy (left) end of the sequence of dark green points of Fig. 7 is an unexpected loop. While the BnGa method introduces resonances and tests to see if they improve the fits to data, this is not one of them. A 


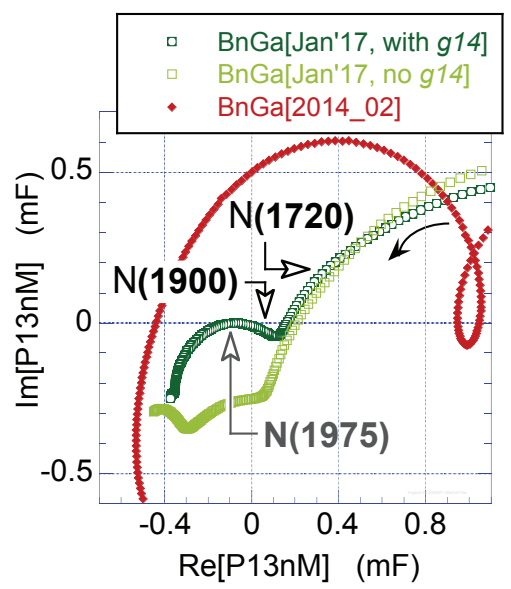

Fig. 7 Argand plot of BnGa PWA solutions for the $P_{13} n M$ multipole from $W=1600$ to $2300 \mathrm{MeV}$. Solid arrow indicates increasing $W$. As in the legend, red diamonds are the on-line version [20], light-green squares are fitted to all previously published data, and dark-green circles augment these with the new $E$ asymmetries from ref. [10]. (See text.)

subsequent BnGa reanalysis of neutron target data finds some evidence for an $N 3 / 2+$ resonance centered at about $W=1975 \mathrm{MeV}$, with couplings of $A_{n}^{1 / 2}=-26 \pm 13$ and $A_{n}^{3 / 2}=-77 \pm 15$, although the energy is poorly constrained. It is quite possible that this is in fact a new $N(1945) 3 / 2+$ resonance, which shows up strongly in hyperon decay channels and had not previously been observed in the $\pi N$ final state [30]. (We list the energy as $1945 \mathrm{MeV}$ in the summary of Table 2 below.)

Finally, in Fig. 8 we show an expanded region of the Argand graphs for the $S_{11} n E$ multipole from both BnGa (left panel) and SAID (right panel) analyses. The four-star $N(1650) 1 / 2$ - resonance generates a counter-clockwise loop in both PWA. In the BnGa analysis, fits that include the $g 14 E$ asymmetry data reveal a second loop which is now associated with an $N(1895) 1 / 2-$ state. While evidence for this resonance had not previously been observed in $\pi N$ channels, strong signals have been reported recently in hyperon decay [30]. The fact that it now manifests itself in a fit to a $\pi^{-} p$ asymmetry echoes the potential of polarization observables.

The SAID $S_{11} n E$ multipole also shows a counter-clockwise rotating phase motion in the region of $W=1895$ (right panel of Fig. 8). As discussed in Section 3.1, in the SAID procedure this comes from fits of a polynomial parametrization of the numerator of Eq. 2 to photoproduction data. It is interesting that this procedure finds the same general phase motion in this partial wave. Nonetheless, since the SAID methodology requires a definitive signal in $\pi N \rightarrow \pi(\eta) N$ reactions, it cannot directly confirm a resonance that couples mainly to $K Y$. 

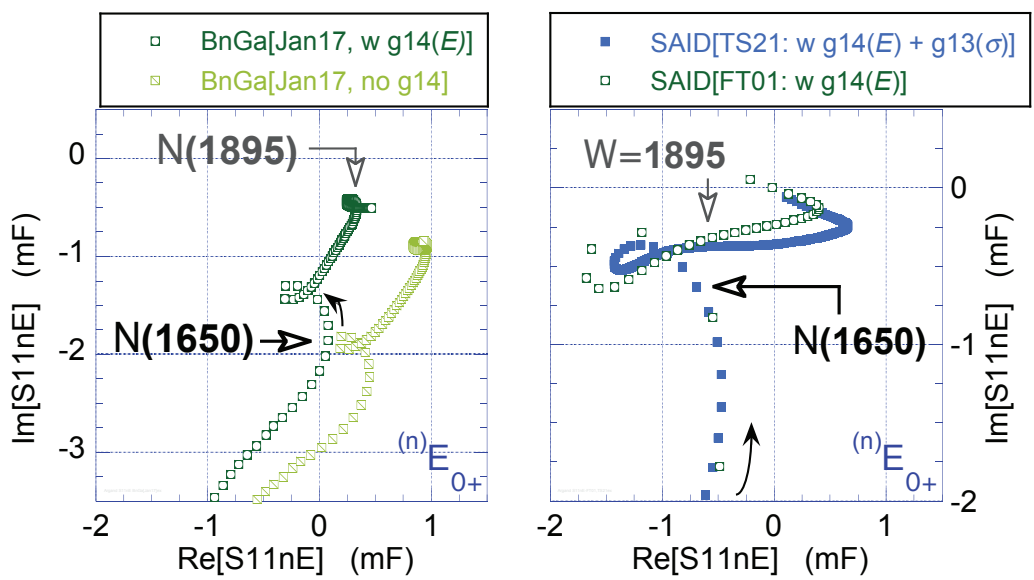

Fig. 8 Argand plots of PWA solutions for the $S_{11} n E$ multipole from $W=1600$ to $2300 \mathrm{MeV}$, with BnGa solutions on the left and SAID on the right. Solid arrows indicates increasing $W$. Dark green hollow-circles were fitted to all data up to and including the $g 14 E$ asymmetries; these PWA result in the solid curves of Fig. 5 [10]. Light green squares on the left are from the BnGa fit that excludes the $g 14 E$ data. The solid blue squares in the right panel are SAID fits that include the new $g 13$ cross section results [28]; this is the same PWA as in the last column of Table 1. (See text.)

\section{Summary}

Direct solutions of the QCD Lagrangian on a lattice have confirmed the Quark Model expectation for large numbers of excited nucleon states, well beyond the modest number that have been identified as resonances in $\pi N$ reactions. The $\pi N \rightarrow \pi N$ process has only four possible observables and present data are severely limited, resulting in under-determined amplitudes. In contrast, meson photoproduction requires eight of sixteen possible observables to determine their spin-dependent amplitudes. Extensive data sets on large numbers of polarization observables have been acquired from new facilities, such as the CLAS detector at Jefferson Lab, which are expected to over-constrain the production amplitudes. Analyses are ongoing. The evaluation of couplings to established resonances is improving and many new $N^{*}$ candidates are emerging. The examples discussed here focus on recent CLAS measurements of the beam-target helicity asymmetry $(E)$ in $\pi^{-} p$ photo-production from (effective) polarized neutrons. With the addition of this single double-polarization observable to the sparse $\gamma n$ data base, signatures emerge from weak resonances that couple strongly to hyperon channels but had not previously been observed in $\pi N$. Other polarization observables are currently under analysis and their results should prove illuminating [31].

Couplings at the electromagnetic vertex are sensitive to the mechanism of resonance excitation. While the photo-couplings to $\Delta^{*}$ resonances are identical for neutron and proton targets, the $\gamma n N^{*}$ and $\gamma p N^{*}$ photo-couplings are different for $I=1 / 2$ states and provide complementary information. A comparison 
Table 2 A comparison of $\gamma n N^{*}$ and $\gamma p N^{*}$ helicity amplitudes from SAID [21,29] and $\mathrm{BnGa}[10,24,25]$ partial wave analyses, for the resonances discussed in the previous sections. Consistent findings of the SAID and BnGa analyses are highlighted as bold text.

\begin{tabular}{lcccc}
\hline$\left(10^{-3} \mathrm{GeV}^{-1 / 2}\right)$ & $A_{n}^{1 / 2}$ & $A_{p}^{1 / 2}$ & $A_{n}^{3 / 2}$ & $A_{p}^{3 / 2}$ \\
\hline$S A I D:$ & $\mathbf{1 5} \pm \mathbf{5}$ & $\mathbf{9 5} \pm \mathbf{2}$ & $+13 \pm 4$ & $-48 \pm 2$ \\
$N(1720) 3 / 2^{+}$ & - & - & & - \\
$N(1895) 1 / 2^{-}$ & - & - & - & - \\
$N(1945) 3 / 2^{+}$ & $-16 \pm 5$ & - & $-\mathbf{3 5} \pm \mathbf{5}$ & - \\
$N(2190) 7 / 2^{-}$ & & & & \\
$B n G a:$ & $-(\mathbf{2 8}+\mathbf{4 0 / - 1 5 )}$ & $+\mathbf{1 1 0} \pm \mathbf{4 5}$ & $\pm(103 \pm 35)$ & $+150 \pm 30$ \\
$N(1720) 3 / 2^{+}$ & $-15 \pm 10$ & $-11 \pm 6$ & & \\
$N(1895) 1 / 2^{-}$ & $-26 \pm 13$ & - & $-77 \pm 15$ & - \\
$N(1945) 3 / 2^{+}$ & $+30 \pm 7$ & $-65 \pm 8$ & $-\mathbf{2 3} \pm \mathbf{8}$ & $+35 \pm 17$ \\
$N(2190) 7 / 2^{-}$ & & & & \\
\hline
\end{tabular}

of the two for the resonances discussed here are summarized in Table 2. While the available published data is still insufficient to remove all ambiguities when determining amplitudes, particularly for neutron reactions, there is agreement between the SAID and BnGa PWA on a number of helicity amplitudes. Given the differences between the two analysis approaches, the agreement is very encouraging.

Acknowledgements We are grateful to Dr. T-S. H. Lee for many fruitful discussions and for his invaluable theoretical studies on the implications of analysis requirements, and to Drs. R.L. Workman, A. Švarc, E. Klempt and A. Sarantsev for many useful discussions and for providing results from their multipole analyses. This work has been supported by the U.S. Department of Energy, Office of Nuclear Physics, under Contract No. DE-AC05-06OR23177, under which Jefferson Science Associates operate Jefferson Laboratory.

\section{References}

1. Bhagwat M.S., Pichowsky M.A., Roberts C.D. and Tandy P.C., Analysis of a quenched lattice-QCD dressed-quark propagator, Phys. Rev. C68 015203 (2003).

2. Capstick S. and Roberts W., Strange decays of nonstrange baryons, Phys. Rev. D 58, 074011 (1998).

3. Bazavov A. et al., Additional Strange Hadrons from QCD Thermodynamics and Strangeness Freezeout in Heavy Ion Collisions, Phys. Rev. Lett. 113, 072001 (2014).

4. Edwards R., J. Dudek, Richards D. and Wallace S., Excited state baryon spectroscopy from lattice QCD, Phys. Rev. D 84074508 (2011).

5. Chaing W-T. and Tabakin F., Completeness rules for spin observables in pseudoscalar meson photoproduction, Phys. Rev. C55, 2054 (1997).

6. Sandorfi A.M., Hoblit S., Kamano H. and Lee T-S.H., Determining pseudoscalar meson photoproduction amplitudes from complete experiments, J. Phys. G38, 053001 (2011).

7. Suzuki N., Juliá-Díaz B., Kamano H., Lee T-S.H., Matsuyama A. and Sato T., Disentangling the Dynamical Origin of $P_{11}$ Nucleon Resonances, Phys. Rev. Lett. 104, 042302 (2010)

8. Park K. et al. (CLAS Collaboration), Measurements of ep $\rightarrow e^{\prime} \pi^{+} n$ at $1.6<W<2.0$ GeV and extraction of nucleon resonance electrocouplings at CLAS, Phys. Rev. C91, 045203 (2015). 
9. Mecking B.A. et al., The CEBAF large acceptance spectrometer (CLAS), Nucl. Inst. Meth. Phys. Res. A503, 513 (2003)

10. Ho D. et al. (CLAS Collaboration), Beam-Target Helicity Asymmetry for $\gamma n \rightarrow \pi^{-} p$ in the $N^{*}$ Resonance Region, Phys. Rev. Lett. 118, 242002 (2017)

11. Sandorfi A.M., Dey B., Sarantsev A.V., Tiator L., and Workman R.L., A Rosetta Stone Relating Conventions In Photo-Meson Partial Wave Analyses, AIP Conf. Proc. 1432, 219 (2012); arXiv:1108.5411v2.

12. Bass C.D et al., A portable cryostat for the cold transfer of polarized solid HD targets: HDice - I, Nucl. Inst. Meth. Phys. Res. A737, 107 (2014).

13. Lowry M.M. et al., A cryostat to hold frozen-spin polarized HD targets in CLAS: HDice - II, Nucl. Inst. Meth. Phys. Res. A815, 31 (2016).

14. Ho D., Measurements of the $E$ Polarization Observable for $\gamma d \rightarrow \pi^{-} p\left(p_{s}\right), \gamma d \rightarrow$ $K^{0} \Lambda\left(p_{s}\right)$, and $\gamma d \rightarrow \pi^{+} \pi^{-} d(0)$ using CLAS g14 data at Jefferson Lab, Ph.D. thesis, Carnegie Mellon University (2015); http://repository.cmu.edu/dissertations/590.

15. Wu J.J., Sato T., and Lee T-S.H., Incoherent pion production in neutrino-deuteron interactions, Phys. Rev. C91, 035203 (2015).

16. Polyzou W.N., Glöckle W., and Witala H., Spin and Dynamics in Relativistic Quantum Theories, Few Body Syst. 56, 395 (2015); Lee T-S.H. (priv. comm.)

17. Giebink D.R., Relativity and spin in one-, two-, and three-body systems, Phys. Rev. C 32, 502 (1985).

18. Nakamura S., contribution to these conference proceedings; (priv. comm.).

19. Workman R.L. et al., GW Institute for Nuclear Studies - Data Analysis Center, http://gwdac.phys.gwu.edu.

20. Nikonov V.A. et al., Bonn-Gatchina Partial Wave Analysis, http://pwa.hiskp.unibonn.de.

21. Workman R.L., Paris M.W., Briscoe W.J. and Strakovsky I.I, Unified Chew-Mandelstam SAID analysis of pion photoproduction data, Phys. Rev. C86, 015202 (2012).

22. Gutz E, et al., High statistics study of the reaction $\gamma p \rightarrow p \pi^{0} \eta$, Eur. Phys. J. A50, 74 (2014)

23. Adlarson P. et al., Measurement of $\pi^{0}$ photoproduction on the proton at MAMI C, Phys. Rev. C92, 024617 (2015), (and references therein).

24. Anisovich A.V. et al., Neutron helicity amplitudes, Phys. Rev. C 96, 055202 (2017).

25. Anisovich A.V. et al., Properties of baryon resonances from a multichannel partial wave analysis, Eur. Phys. J. A 48, 15 (2012).

26. Patrignani C. et al. (PDG), Review of Particle Physics, Chin. Phys. C40, 100001 (2016).

27. Workman R.L., Briscoe W.J., Paris M.W. and Strakovsky I.I, Updated SAID analysis of pion photoproduction data, Phys. Rev. C85, 025201 (2012).

28. Mattione P.T. et al. (CLAS Collaboration), Differential cross section measurements for $\gamma n \rightarrow \pi^{-} p$ above the first nucleon resonance region, Phys. Rev. C96, 035204 (2017); Mattione P., contribution to these Proceedings.

29. Workman R.L. and Švarc A., priv. comm. (2017).

30. Anisovich A.V. et al., Strong Evidence for Nucleon Resonances near $1900 \mathrm{MeV}$, Phys. Rev. Lett. 119062004 (2017).

31. $\mathrm{Lu} \mathrm{H}$., contribution to these Proceedings. 